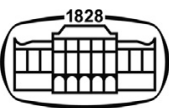

AKADÉMIAI KIADÓ

\title{
Ophthalmic signs and complications of the COVID-19 infection
}

\author{
Z. Z. NAGY ${ }^{1,2^{*}}$
}

\section{Developments in Health Sciences}

$3(2020) 4,79-82$

DOI:

10.1556/2066.2021.40001

(c) 2021 The Author(s)

\section{EDITORIAL}

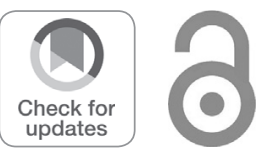

Corresponding author.

E-mail: zoltan.nagy100@gmail.com
${ }^{1}$ Department of Ophthalmology, Faculty of Medicine, Semmelweis University, Budapest, Hungary

${ }^{2}$ Department of Clinical Ophthalmology, Faculty of Health Sciences, Semmelweis University, Budapest, Hungary

\section{ABSTRACT}

Coronavirus-19 infection caused a mysterious pandemic worldwide. Some people experienced flu-like symptoms, while others have died due to pulmonary complications. Besides droplet spread, other routes of infection started to be suspected, such as through eye contact. During the first phase of the pandemic, pulmonary symptoms were in focus, later other signs and symptoms were also published. Eyelid, anterior and posterior segment symptoms, neuro-ophthalmic complications, and orbital problems related to COVID-19 infections are discussed in this article. It is important to detect the serious signs and symptoms to prevent late, sight threatening complications of COVID-19 infection.

\section{KEYWORDS}

Coronavirus-19, pandemic, ophthalmology, ophthalmic complications

\section{INTRODUCTION}

The first phase of Coronavirus-19 pandemic started at the end of 2019 in the city of Wuhan, China. Since then, humankind experienced the second and third phases of the viral infection, which grew into a worldwide pandemic. Millions of people became infected and the death toll is exponentially increasing. Vaccination might offer a solution against the spreading of the virus. Also, the course of the disease seems also milder following vaccination against COVID19. Many publications describe the pulmonary, sensory nervous system, and general symptoms.

There are several reports dealing with ophthalmic, usually non-specific signs and symptoms. The route of infection is usually by droplet inhalation, although conjunctival route has been described and debated as well [1]. Recently more and more neuroophthalmological complications are published in the literature. Coronavirus infection is still a mystery regarding the symptoms and severity of the infection. In some people it is very mild, while some people may die due to pulmonary complications. Earlier obese, diabetic, hypertensive elderly patients were in danger. With the introduction of specific vaccinations, the death incidence decreased among elderly people. On the other hand, without prior vaccination a specific increase could be observed in the younger generations.

\section{Reported ophthalmic signs}

Follicular conjunctivitis. Many patients showed conjunctival redness during the acute phase of the COVID-19 infection. In many cases the hand-eye contact could be excluded from history. During the middle phase of the disease, follicular conjunctivitis was reported in some patients. The conjunctival swab was positive [2], thereafter positivity disappeared. In another publication follicular conjunctivitis was found four weeks after the COVID infection. The patients had hypertension, diabetes mellitus, and asthma [3].

Chemosis. Chemosis (oedematous change of the conjunctiva) is often reported in intensive care units (ICUs). This condition is usually self-limited and resolves shortly after the patient gets better. 
Specific conjunctivitis in children. In children, a specific conjunctivitis, such as acute subconjunctival haemorrhage and injection is usually reported together with Kawasaki syndrome. This is a multisystem inflammatory process, including vasculitis, iridocyclitis, punctate keratitis, vitreous opacities, and specific conjunctivitis. Treatment should aim to treat systemic inflammation with corticosteroids, antibiotic topical drops in intravenous immunoglobulins [4].

Haemorrhagic and pseudomembranous conjunctivitis. Navel reported a patient who developed haemorrhagic conjunctivitis with pseudomembrane formation almost three weeks after the onset of COVID-19 infection in the ICU. Symptoms were treated with antibiotic (azithromycin) and steroid (dexamethasone) drops and resolved gradually without severe sequelae [5].

Episcleritis/scleritis. Some cases of scleritis/episcleritis have been reported since the outbreak. Patients complain of foreign body sensation together with conjunctival and episcleral vessel congestion. The congestion could be blanched with topical phenylephrine $[6,7]$.

Hyperaemic eyelids. During COVID-19 infection in some cases lid margin telangiectasia or hyperaemia was observed, causing symptoms of blepharitis. During eyelid and ocular surface problems the following symptoms might appear: discharge, redness, tearing, and dryness of ocular surface with consequent visual deterioration. Besides foreign body sensation, burning, itching, photophobia, crusted eyelashes, chemosis, follicular conjunctivitis might be present as well [8].

Central retinal artery occlusion (CRAO). In the central nervous system and within the retina, vascular, inflammatory, and neuronal changes might be caused by the COVID-19 infection. Central retinal artery occlusion is an acute vision threatening entity with bad prognosis. In patients with CRAO elevated inflammatory markers, IL-6, C reactive protein (CRP), fibrinogen and D-dimer were reported [9].

Central retinal vein occlusion (CRVO). In severe cases CRVO might appear. The course and consequences are greatly similar to non-COVID-CRVO-s. Elevated prothrombin time (PT), activated partial thromboplastin time (aPTT), elevated fibrinogen and cytokine levels were found. Hypertension, diabetes, and hyperlipidaemia might also be concomitant with the COVID infection. Intubation and forced respiration in patients with pneumonia might trigger the endothelial cells to start extrinsic coagulation cascade, resulting in CRVO within the central vein of the eye [10].

Acute macular neuroretinopathy (AMN). The cause of AMN is still unknown, but it is believed that in $50 \%$ of the cases there was a flu-like infection or other respiratory disease behind AMN symptoms. Painless, acute loss of vision, paracentral scotoma, loss of colour vision are frequent symptoms. Roth spots in the retina were also observed in some patients. OCT angiography revealed reduced blood flow in all capillary plexuses (superficial, intermediate, and deep plexus layers) [11].

Vitritis. Vitritis means an opacity within the vitreous, caused by disorganised collagen fibers and inflammatory cells. Usually, patients complain of blurred vision and opacities which might change their positions with eye movements. Vitritis might be part of the general inflammatory changes within the eye due to COVID infection [12]. Other entities such as Herpes simplex, Cytomegalovirus, syphilis, toxoplasma, and toxocara infections should be excluded.

Acute retinal necrosis (ARN). Usually, patients with ARN are in an immunocompromised state, having haematologic disorders such as B cell lymphoma or SLE (systemic lupus erythematosus). It is assumed that COVID-19 infection might attack the blood-retinal barrier causing higher inflammatory response within the retina [13].

Serpiginous choroiditis (SC). SC is a rare idiopathic bilateral inflammatory disorder of the choroid and retina, with geographic destruction of the retinal pigment epithelium, retina, and choroid. It is characterised by extensive loss of retinal pigment epithelium and destruction of the overlying retina with similar shape. The disease leaves scars within the retina with loss of visual function of pigment epithelium and photoreceptor cells. Patients complain of blurred vision and central or paracentral scotomas. The presumed mechanism in COVID-19 infection is local inflammatory processes [14].

\section{Neuro-ophthalmic conditions}

During the COVID-19 pandemic in 35\% of the patients some neurological disorders have been described, such as: headache, anosmia, hypogeusia, dizziness, ischaemic stroke, visual deterioration, and Guillain-Barré syndrome. In the background of neurological manifestations, the neurotropism of the COVID virus is suspected.

Papillophlebitis. In papillophlebitis a unilateral and painless decreased visual acuity bothers the patient. Visual field examination reveals an enlarged blind spot. Fundoscopy usually shows tortuous, dilated blood vessels with disc oedema, cotton wool spots, and frequently macular oedema as well. The course of the disease is usually favourable, but in one-third of the cases ischaemic retinal mechanisms causing central venous occlusion and secondary neovascular glaucoma occur. During COVID-19 infection, blood coagulation might change together with the cytokine storm, causing vision problems [15].

Optic neuritis. Optic neuritis was found in several patients usually one to two weeks after COVID infection. Patients presented with painful visual deterioration, RAPD (relative afferent pupillary defect) in the eye with worse visual acuity, visual field defect, and optic nerve enlargement diagnosed with MRI [16]. The MOG (anti-myelin oligodendrocyte glycoprotein) was positive in the reported cases, although viral and immunological diagnostics did not reveal any 
specific pathology. MRI brain imaging was also not specific. Systemic corticosteroid treatment helped to restore vision and resolve the optic nerve head oedema. It is presumed that in the future demyelinating diseases might show an increase in incidence due to viral trigger effects.

Adie's tonic pupil. Adie syndrome includes neurological disorders characterised by tonically dilated pupil, which does not or very slowly constrict to light, but shows a more definite answer to accommodation. It might be associated with rheumatological alteration such as absent knee or ankle reflexes and loss of sweating ability. The presumed cause is a damage to the postganglionic fibers of the parasympathetic innervation of the eye. It starts usually with a viral or bacterial infection that causes inflammation and affects the pupil of the eye and the autonomic nervous system. Adie's tonic pupil was also observed in COVID infected patients, starting with pain behind the eye and reading difficulties. The pupil was hypersensitive to $0.1 \%$ pilocarpin drops, which supported the diagnosis [17].

Miller-Fisher syndrome (MFS). MFS characterised by acute onset of ataxia, diminishing or loss of tendon reflexes, and ophthalmoplegia. Patients revealed acute symptoms of diplopia, the 6th nerve was most frequently affected, followed by the 3rd cranial nerve; cranial nerve palsies were selflimiting disorders [18]. Facial nerve palsy was also published. MFS cases responded well to IV immunoglobulin treatment.

Neurogenic ptosis. Assine reported an acute onset bilateral ptosis with other neurological signs, about three weeks following COVID infection. Immunoglobulin therapy was found to be effective [19].

Cerebrovascular accident (CVA). Acute visual loss with cerebrovascular accident might happen from the procoagulant state during COVID-19 infections. In patients with diabetes, obesity, or SLE the danger of CVA is higher compared to healthy individuals [20].

\section{Orbital complications}

Dacryoadenitis. During the early phase of COVID infection, the virus can travel through the lacrimal pathway to the lacrimal gland, which will enlarge, and haematological spread is also suspected. After the acute phase and immunological response, inflammation of the lacrimal gland might also be triggered [21].

Retro-orbital pain. A highly non-specific symptom is the retro-orbital pain which might be quite severe in patients with COVID-19 infections [22].

Orbital cellulitis and sinusitis. In some patients acute onset of progressive orbital swelling was detected, in the background there was no chronic sinus disease [23].

Mucormycosis. Mucormycosis is a life-threatening opportunistic infection, which was diagnosed in some patients infected with COVID-19 virus. Usually, the patients are immunocompromised and have other comorbidities, like diabetes, weak pulmonary function, and use of immunosuppressive therapy. The COVID-19 mucormycosis is not different from other cases of mucormycosis [24].

Orbital histiocytic lesion. An elderly patient was reported with bilateral proptosis, eyelid swelling, enlarged lacrimal glands, orbital mass, cervical and axillary mediastinal lymphadenopathy. Biopsy revealed benign histiocytic lesion. During COVID-19 infection a compromised immune system was presumed in pathophysiology [25].

\section{CONCLUSIONS}

The Coronavirus-19 infection can affect, beside the respiratory system, the central and peripheral nervous systems as well. Ophthalmic symptoms seemed to be rare at the first phase of the pandemic, but during the next phases more and more ophthalmic and neuroophthalmic disorders have been reported. Most of them are not specifically characteristic to the Coronavirus-19 infection, however, they might affect longterm visual functions. Due to life-threatening pulmonary complications at the intensive care units, ophthalmic assessment does not get into the focus of attention. Together with the neuroophthalmic complications a thoughtful ophthalmic assessment may help to prevent undesired ophthalmic sequelae. Since post-COVID complaints might also affect ophthalmologists, specialists from different fields should communicate with each other to achieve the best post-COVID health gain for the patients.

\section{ABOUT THE AUTHOR}

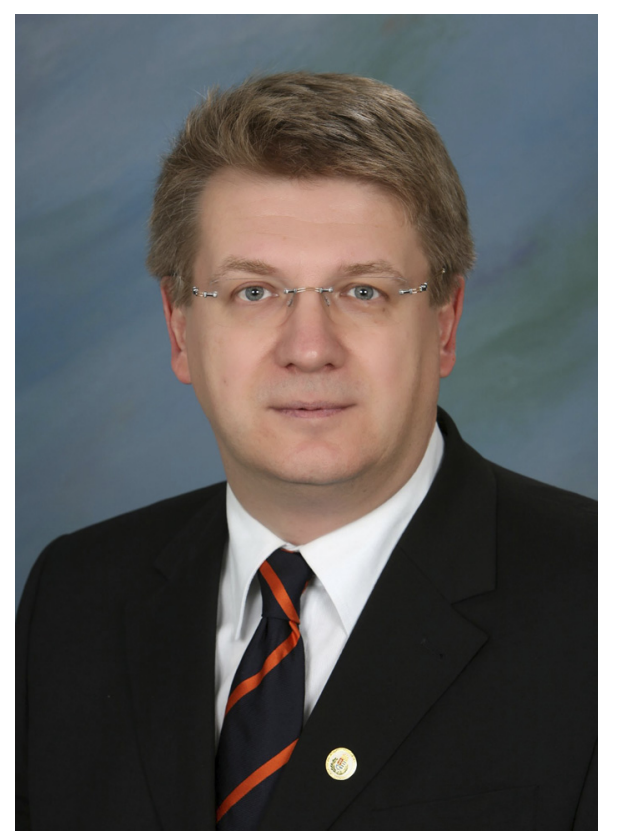

Zoltán Zsolt Nagy, med.habil, DSc, FEBO

Editor-in-Chief 


\section{REFERENCES}

1. Zhou Y, Zeng Y, Tong Y, Chen C. Ophthalmologic evidence against the interpersonal transmission of 2019 novel coronavirus through conjunctiva. medRxiv. https://doi.org/10.1101/2020.02.11. 20021956.

2. Chen L, Deng C, Chen X, Zhang X, Chen B, Yu H, et al. Ocular manifestations and clinical characteristics of 535 cases of COVID-19 in Wuhan, China: a cross-sectional study. Acta Ophthalmol 2020;98:951-9. https://doi.org/10.1111/aos.14472.

3. Nayak B, Poddar C, Panigrahi MK, Tripathy S, Mishra B. Late manifestation of follicular conjunctivitis in ventilated patient following COVID-19 positive severe pneumonia. Indian J Ophthalmol 2020;68:1675-7. https://doi.org/10.4103/ijo.IJO_1682_20.

4. Danthuluri V, Grant MB. Update and recommendations for ocular manifestations of COVID-19 in adults and children. A narrative review. Ophthalmol Ther 2020;9:853-75. https://doi.org/10.1007/ s40123-020-00310-5.

5. Navel V, Chiambaretta F, Dutheil F. Haemorrhagic conjunctivitis with pseudomembranous related to SARS-CoV-2. Am J Ophthalmol Case Rep 2020;19:100735. https://doi.org/10.1016/j.ajoc. 2020.100735 .

6. Otaif W, Al Somali AI, Habash A. Episcleritis as a possible presenting sign of the novel coronavirus disease: a case report. Am J Ophthalmol Case Rep 2020;20:100917. https://doi.org/10.1016/j. ajoc.2020.100917.

7. Zhang X, Chen X, Chen L, Deng C, Zou X, Liu W, et al. The evidence of SARS-CoV-2 infection on ocular surface. Ocul Surf 2020; 18:360-2. https://doi.org/10.1016/j.jtos.2020.03.010.

8. Meduri A, Oliverio GW, Mancuso G, Giuffrida A, Guarneri C, Venanzi Rullo E, et al. Ocular surface manifestation of COVID-19 and tear film analysis. Sci Rep 2020;20178. https://doi.org/10.1038/ s41598-020-77194-9.

9. Acharya S, Diamond M, Anwar S, Glaser A, Tyagi P. Unique case of central retinal artery occlusion secondary to COVID-19 disease. IDCases 2020;21:e0086. https://doi.org/10.1016/j.idcr.2020. e00867.

10. Invernizzi A, Pellegrini $M$, Messenio D, Cereda M, Olivieri $P$, Brambilla AM, et al. Impending central retinal vein occlusion in a patient with coronavirus disease 2019 (COVID-19). Ocul Immunol Inflamm 2020;28:1290-2. https://doi.org/10.1080/09273948.2020. 1807023.

11. Zamani G, Ataei Azimi S, Aminizadeh A, Shams Abadi E, Kamandi $\mathrm{M}$, Mortazi $\mathrm{H}$, et al. Acute macular neuroretinopathy in a patient with acute myeloid leukemia and decreased by COVID-19: a case report. J Ophthalmol Inflamm Infect 2020;10:39. https://doi.org/10. 1186/s12348-020-00231-1.

12. Zago Filho LA, Lima LH, Melo GB, Zett C, Farah ME. Vitritis and outer retinal abnormalities in a patient with COVID-19. Ocul
Immunol Inflamm 2020;28:1298-300. https://doi.org/10.1080/ 09273948.2020.1821898.

13. Gupta A, Dixit B, Stramulas K, Akshikar R. Atypical bilateral acute retinal necrosis in a coronavirus disease 2019 positive immunsuppressed patient. Eur J Ophthalmol 2020:11206721097491. https:// doi.org/10.1177/1120672120974941.

14. Providência J, Fonseca C, Henriques F, Proença R. Serpiginous choroiditis presenting after SARS-CoV-2 infection: a new immunological trigger? Eur J Ophthalmol 2020:1120672120977817. https://doi.org/10.1177/1120672120977817.

15. Insausti-Garcia A, Reche-Sainz JA, Ruiz-Arranz C, Vazquez AL, Ferro-Osuna M. Papillophlebitis in a COVID-19 patient: inflammation and hypercoagulable state. Eur J Ophthalmol 2020: 1120672120947591. https://doi.org/10.1177/1120672120947591.

16. Ortiz-Seller A, Martinez Costa L, Hernandez-Pons A, Valls Pascual E, Solves Alemany A, Albert-Fort M. Ophthalmic and neuroophthalmic manifestations of coronavirus disease 2019 (COVID-19). Ocul Immunol Inflamm 2020;28:1285-9. https://doi.org/10.1080/ 09273948.2020.1817497.

17. Tisdale AK, Chwalisz BK. Neuro-ophthalmic manifestations of coronavirus disease 19. Curr Opin Ophthalmol 2020;31:489-94. https://doi.org/10.1097/ICU.0000000000000707.

18. Gutiérrez-Ortiz C, Méndez-Guerrero A, Rodrigo-Rey S, San PedroMurillo E, Bermejo-Guerrero L, Gordo-Mañas R, et al. Miller Fisher syndrome and polyneuritis cranialis in COVID-19. Neurology 2020; 95:e601-5. https://doi.org/10.1212/WNL.0000000000009619.

19. Huber M, Rogozinski S, Puppe W, Framme C, Höglinger G, Hufendiek K, et al. Postinfectious onset of myasthenia gravis in a COVID-19 patient. Fron Neurol 2020;11:576153. https://doi.org/ 10.3389/fneur.2020.576153.

20. Yang Y, Qidway U, Burton BJ, Canepa C. Bilateral vertical supranuclear gaze palsy following unilateral midbrain infarct. BMJ Case Rep 2020;13:e238422. https://doi.org/10.1136/bcr-2020-238422.

21. Martinez Diaz M, Copete Piqueras S, Blanco Marchite C, Vahdani $\mathrm{K}$. Acute dacryoadenitis in a patient with SARS-CoV-2 infection. Orbit 2021:1-4. https://doi.org/10.1080/01676830.2020.1867193.

22. Rui W, Hsu SY, Tsai HL, Chen CT, Tseng CP, Chen WT. COVID-19 mimicking dengue fever with the initial manifestation of retro-orbital pain - a rare case. J Formos Med Assoc 2020;119: 1715-6. https://doi.org/10.1016/j.jfma.2020.05.039.

23. Turbin RE, Wawrzusin PJ, Sakla NM, Traba CM, Wong KG, Mirani N, et al. Orbital cellulitis, sinusitis and intracranial abnormalities in two adolescents with COVID-19. Orbit 2020;39:305-10. https://doi.org/10.1080/01676830.2020.1768560.

24. Werthman-Ehrenreich A. Mucormycosis with orbital compartment syndrome in a patient with COVID-19. Am J Emerg Med 2021;42: 264.e5-8. https://doi.org/10.1016/j.ajem.2020.09.032.

25. Vemuganti GK, Naik MN, Honavar SG. Rosai dorfman disease of the orbit. J Hematol Oncol 2008;1:7. https://doi.org/10.1186/17568722-1-7.

\footnotetext{
Open Access. This is an open-access article distributed under the terms of the Creative Commons Attribution-NonCommercial 4.0 International License (https:// creativecommons.org/licenses/by-nc/4.0/), which permits unrestricted use, distribution, and reproduction in any medium for non-commercial purposes, provided the original author and source are credited, a link to the CC License is provided, and changes - if any - are indicated.
} 